\title{
Antibacterial effect of silver nanoparticles prepared in bipolymers at moderate temperature
}

\begin{abstract}
The purpose of this study was to investigate the antibacterial effect of silver nanoparticles in chitosan-poly(ethylene glycol) suspension. The silver nanoparticles (AgNPs) were prepared by use of an environmentally benign method from chitosan (Cts) and poly(ethylene glycol) (PEG) at moderate temperature and with stirring for different times. Silver nitrate (AgNO3) was used as the metal precursor and Cts and PEG were used as solid support and polymeric stabilizer, respectively. The antibacterial activity of silver-chitosan-poly(ethylene glycol) nanocomposites (Ag-Cts-PEG NCs) against Staphylococcus aureus, Micrococcus luteum, Pseudomonas aeruginosa, and Escherichia coli was tested by use of the Mueller-Hinton agar disk-diffusion method. Formation of AgNPs was determined by UV-visible spectroscopy; surface plasmon absorption maxima were observed at $415-430 \mathrm{~nm}$ in the UV-visible spectrum. The peaks in the XRD pattern confirmed that the AgNPs had a face-centered cubic structure; peaks of contaminated crystalline phases were not observed. Transmission electron microscopy (TEM) revealed that the AgNPs synthesized were spherical. The optimum stirring time for synthesis of the smallest particle size (mean diameter $5.50 \mathrm{~nm}$ ) was $12 \mathrm{~h}$. The AgNPs in Cts-PEG were effective against all the bacteria tested. Higher antibacterial activity was observed for AgNPs with smaller size. These results suggest that AgNPs can be used as an effective inhibitor of bacteria and can be used in medical applications. These results also suggest that AgNPs were successfully synthesized in Cts-PEG suspension at moderate temperature with different stirring times.
\end{abstract}

Keyword: Antibacterial activity; Silver nanoparticles; Bipolymers; Nanocomposites; Moderate temperature 